\title{
Embedded interdigital transducers for high-frequency surface acoustic waves on GaAs
}

\author{
M. M. de Lima, Jr., W. Seidel, H. Kostial, and P. V. Santos ${ }^{a)}$ \\ Paul-Drude-Institut für Festkorperelektronik, Hausvogteiplatz, 5-7, 10117 Berlin, Germany
}

(Received 27 April 2004; accepted 16 June 2004)

\begin{abstract}
We investigate high-performance, high-frequency interdigital transducers (IDTs) for the generation of surface acoustic waves (SAWs) on GaAs substrates, where the metal fingers are embedded in the substrate. We demonstrate that the acoustic reflections and the scattering of the surface modes into the substrate become considerably reduced in these transducers, leading to an increased output power. The finger embedding process is particularly relevant for the generation of powerful beams of high-frequency SAWs on weak piezoelectric substrates (such as most of the semiconducting materials) using long IDTs. We also show that the reflection reduction is important for the design of focusing single-finger IDTs, since it minimizes the effects of the finger grating on the angular dependence of the phase velocity. (C) 2004 American Institute of Physics. [DOI: 10.1063/1.1782961]
\end{abstract}

\section{INTRODUCTION}

Surface acoustic waves (SAWs) are vibrations propagating along the surface of a material, which have been traditionally used in electronic signal processing and in acoustooptic modulation. Most of these applications are based on strong piezoelectric substrates, where SAWs can be efficiently generated by interdigital transducers (IDTs) consisting of an array of metal fingers deposited on the surface of the sample, as illustrated in Fig. 1(a). The electric field induced by a radio-frequency (rf) voltage $V_{\text {rf }}$ applied to the fingers generates a strain field in the material through the piezoelectric effect. The frequency of the rf voltage required for the excitation of the SAW mode is given by the ratio between the SAW propagation velocity $\left(v_{\text {SAW }}\right)$ and the SAW wavelength $\left(\lambda_{\mathrm{SAW}}\right)$. For the IDT of the single-finger (SF) type displayed in Fig. 1(a), $\lambda_{\text {SAW }}$ is equal to twice the periodicity of the metal grating $\left(\lambda_{\mathrm{IDT}}\right)$.

Nowadays, there is substantial interest in using highfrequency and high-power SAWs to modulate the mechanical, electronic, and optical properties of low-dimensional structures, many of them based on semiconductor materials. Examples of these applications include the acoustically induced charge transport, ${ }^{1}$ light storage, ${ }^{2}$ modulation of photonic structures ${ }^{3}$ and optical cavities, ${ }^{4}$ and the driving of micromechanical systems. 5 , As in conventional piezoelectric insulators, the SAWs can be conveniently generated in piezoelectric semiconductors (such as, for instance, the III-V materials) using IDTs. Most of the conventional semiconductors (such as the GaAs-based materials), however, have very weak piezoelectric coupling coefficients. The generation of strong SAW fields on these materials thus requires IDTs with a large number of fingers, through which the SAW has to propagate before leaving the IDT. Since each finger has a finite probability of reflecting and scattering the SAW beam into the substrate, SAW losses in long IDTs due to reflection and scattering become an important issue.

\footnotetext{
a) Author to whom correspondence should be addressed; electronic mail: santos@pdi-berlin.de
}

The acoustic reflection effects become particularly strong in SF IDTs, where the period of the metal grating fulfills the Bragg condition for constructive reflection of the SAW beam. The Bragg reflections turn these IDTs into a standing-wave acoustic cavity with a high acoustic amplitude inside the transducers. In addition, the multiple reflections at the metal fingers enhance the scattering of the SAW into bulk modes, thus reducing the SAW power delivered outside the IDT. Furthermore, the reflection also affects the relationship between the SAW frequency and wavelength, and thus the frequency response of the IDTs.

In this paper, we investigate the generation of SAW beams on GaAs substrates using single-finger IDTs with metal fingers embedded into the substrate, as illustrated in Fig. 1(b). Such a process has recently been proposed to control the acoustic reflection induced by metal gratings, but experimental results confirming the performance enhancement have not been presented. ${ }^{7}$ We describe a simple process for the fabrication of these IDTs, where the finger embedding

(a)

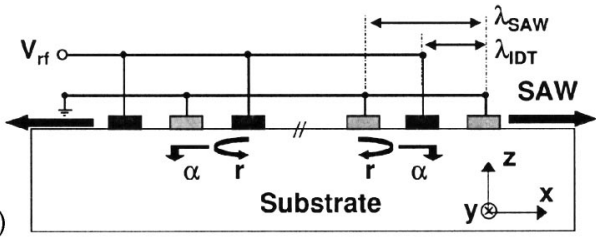

(b)

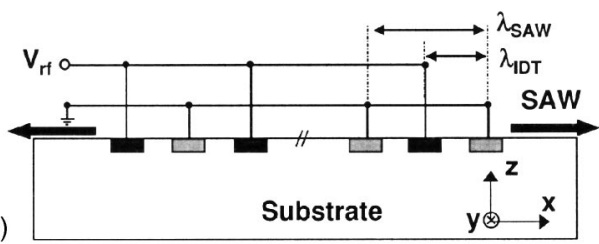

FIG. 1. Schematic diagram of single-finger (SF) interdigital transducers (IDTs) with (a) normal and (b) embedded metal gratings. The fingers are oriented along the $y$ direction. $\lambda_{\mathrm{IDT}}$ and $\lambda_{\mathrm{SAW}}$ denote the period of the metal grating and of the acoustic wave, respectively, while $V_{\text {rf }}$ is the radiofrequency voltage applied to the IDT. $r$ is the amplitude reflection coefficient per unit length of the acoustic wave at the finger grating and $\alpha$ denotes the probability of scattering (also per unit length) of the SAW into bulk modes. 
is achieved by an etching step prior to the deposition of the IDT metal layer, which is fully compatible with the conventional SAW technology. By measuring the acoustic field within and outside the IDTs, we show that the embedding process considerably reduces the reflection at the fingers, in agreement with the predictions of Ref. 7. As a consequence, the output SAW power and the frequency bandwidth increases as compared with conventional single-finger IDTs. The embedding process is particularly important for the generation of high-frequency, high-power SAWs on GaAs, which require long IDTs. Finally, the reduced influence of the fingers on SAW propagation facilitates the design of focusing IDTs, which rely on a precise knowledge of the angular dependence of the SAW propagation velocity. In fact, we show that the performance of focusing IDTs with embedded-finger shapes calculated taking into account the acoustic anisotropy of the substrate is considerably higher than in unembedded control samples.

We start in Sec. II with a discussion of the effects of the metal finger grating on the propagation properties of SAWs based on calculations of the SAW dispersion relation using numerical methods. The procedure employed for the fabrication of embedded-finger IDTs is described in Sec. III. Section IV presents a detailed comparison of the performance of embedded-finger and normal IDTs. The main conclusions are summarized in Sec. V.

\section{SAW REFLECTION AT METAL GRATINGS}

\section{A. Reflection coefficient}

The effects of the SAW reflection caused by the fingers on the acoustic power $P$ delivered by an IDT can be quantified by defining an amplitude reflection coefficient $r$ per unit length, as is illustrated in Fig. 1. $P$ also depends on the scattering of the surface mode into bulk ones upon reflection at the fingers, which is accounted for by the scattering probability per unit length $\alpha$. If $L_{\mathrm{IDT}}$ denotes the length of the IDT, the acoustic power delivered by the device becomes

$$
\begin{aligned}
& P\left(L_{\mathrm{IDT}}\right) \approx\left(g L_{\mathrm{IDT}}\right)^{2}\left[1-(\alpha+r) L_{\mathrm{IDT}}\right] \\
& \text { for }(\alpha+r) L_{\mathrm{IDT}} \ll 1 \\
& \approx \frac{g^{2}\left[1-e^{\left.-(\alpha+r) L_{\mathrm{IDT}}\right]^{2}}\right.}{(\alpha+r)^{2}} \text { for }(\alpha+r) L_{\mathrm{IDT}} \gg 1,
\end{aligned}
$$

where $g$ denotes the SAW generation rate per unit length of the IDT. Equation (1) indicates that reflection effects become significant, when $L_{\mathrm{IDT}}$ is comparable to the effective scattering length $L_{\mathrm{eff}}=1 /(\alpha+r)$. Equation (2) expresses the fact that in a long IDT only a section of maximum length $L_{\text {eff }}$ contributes to $P\left(L_{\mathrm{IDT}}\right)$, delivering an acoustic power $P_{\max }=\left[g L_{\mathrm{eff}}\right]^{2}$. The acoustic power produced by the remaining sections is lost through scattering into bulk modes.

Two approaches have been proposed to reduce the losses caused by acoustic reflections within the IDT. In the first and the most straightforward one, the acoustic mismatch between the metal-free and the metal-covered regions is reduced by an appropriate selection of the metal acoustic properties. The mismatch can normally be minimized by using a low-density metal for the fingers, so as to reduce the mass loading effect. As an example, IDTs on GaAs substrates with aluminum fingers exhibit lower reflection levels than similar devices employing fingers out of gold, which has a much higher mass density.

In the second approach, the geometry of the metal fingers within a SAW period is designed in such a way as to induce a destructive interference of the beams reflected at successive fingers. ${ }^{8}$ This approach normally requires more than two fingers per SAW wavelength as well as a precise control of the finger width and finger separation. A wellknown example is the split-finger configuration, where the metal grating is changed into a second-order one at the SAW wavelength by doubling the number of fingers per period. For the same operation frequency, however, this approach requires finger widths considerably smaller than those for SF IDTs. The reduced feature size is a serious constraint for the lithographic techniques employed for the fabrication of highfrequency IDTs.

The procedure investigated here [see Fig. 1(b)] follows the first of these approaches, where the acoustic mismatch is reduced by embedding the fingers into the substrate. ${ }^{7}$ The reflection of surface waves due to the presence of metallic stripes has been described using simple models based on the elastic properties ${ }^{9}$ and on the charge distribution in the metallic stripe. ${ }^{10}$ In the following section, we present calculations of the dispersion relation for surface elastic modes in conventional and in embedded-finger SF IDTs performed by taking advantage of the periodicity of the fingers. In this approach, the SAW reflection coefficient is estimated from the width of the stop band introduced by the Bragg reflection on the finger grating.

\section{B. Dispersion relation for SAWs}

The acoustic reflection at the interface between two isotropic layers is governed by the difference in acoustic impedances $Z_{i}=\rho_{i} v_{i}=\sqrt{\rho_{i} c_{i}}$ between the two materials (denoted by the subscript $i=1,2) .{ }^{11}$ Here, $\rho_{i}, c_{i}$, and $v_{i}$ denote the density, the elastic constant, and the acoustic velocity, respectively. $Z_{i}$ plays for acoustic waves a role analogous to the refractive index for optic waves. In a periodic structure composed of two layers per period, the dispersion relation for elastic waves can be represented within a mini-Brillouin zone with wave vectors $k$ in the range $-\pi / \lambda_{\mathrm{IDT}}<k<\pi / \lambda_{\mathrm{IDT}}$, where $\lambda_{\text {IDT }}$ denotes the period. Constructive Bragg reflection at the interface between the layers creates stop bands at the boundary of the mini-Brillouin zone (i.e., for a wave vector $k$ $=\pi / \lambda_{\mathrm{IDT}}$ and center frequency $\omega_{c} \approx \bar{v} k$ ). Here, $\bar{v}$ denotes the average acoustic velocity. The width $\Delta \omega$ of the stop band and the acoustic reflection per period $r \lambda_{\text {IDT }}$ within the stop band are determined by the ratio $F=Z_{1} / Z_{2}$ between the acoustic impedances of the two layers. For $F$ close to 1 , it can be shown that the relative ratio $\Delta \omega / \omega_{c}$ becomes $\Delta \omega / \omega_{c} \approx(1$ $-F) /(1+F)$. The reflection coefficient within the stop band depends on frequency and reaches its maximum value at $\omega_{c}$. In the approximation $F \approx 1$, the SAW reflection coefficient at 
the fingers is given simply by $|1-F| \lambda_{\text {IDT }}$. These approximations will be used below to estimate the reflection coefficients of SAW on metal gratings.

The concepts presented in the previous paragraph apply, strictly speaking, only for periodic one-dimensional layer structures. They can, however, be extended to SAWs propagating on a substrate covered by a metal grating, such as the SF IDT of Fig. 1. The two layers are replaced, in this case, by the regions of the IDT without and with the metallization. The effective acoustic impedance $Z_{\text {eff }}$ depends now on the elastic constants and densities of the layers and on the depth distribution of the SAW acoustic field. The SAW mode excited by the IDTs has a wave vector $k_{\mathrm{SAW}}=2 \pi / \lambda_{\mathrm{SAW}}$ $=\pi / \lambda_{\text {IDT }}$ corresponding to the boundary of the miniBrillouin zone. This mode is thus expected to undergo strong Bragg reflections at the metal grating.

In order to quantify the reflection effects, we performed numerical calculations of the SAW dispersion in the metal gratings. The calculations were carried out in two steps, as described in the Appendix of Ref. 6. First, the differential equation for the propagation of acoustic modes was solved for a specific angular frequency $\omega_{\mathrm{SAW}}$ and wave vector $k_{\mathrm{SAW}} \cdot{ }^{11}$ We then sought for solutions satisfying the mechanical and electrical boundary conditions at the surface. In order to take into account the effects of the metal gratings on the SAW excitation, one has to include the spatial variation of materials parameters (such as the elastic constants, mass density, as well as the piezoelectric and dielectric tensors) along the depth and along the SAW propagation direction. For this purpose, we followed the procedure outlined in Refs. 12 and 13, which expresses these parameters in terms of a Fourier series with the number of terms truncated to $n_{G}$. In general, the $n_{G}$ required to reproduce the material parameters in the grating region increases with the thickness $d_{m}$ of the metal fingers. For $d_{m}<10 \%$ of $\lambda_{\mathrm{SAW}}$, we verified that the frequency of the surface eigenmodes do not change significantly for $n_{G}>17$. The results presented here were obtained using $n_{G}=25$.

The open symbols in Fig. 2 display the dispersion relation for surface modes propagating along $\langle 110\rangle$ directions of a (001) GaAs surface covered by the Al metal grating of a conventional SF IDT [see Fig. 1(a)]. Al was used in the calculations since it is a metal commonly used for IDT metallization. The thickness $d_{m}$ of the $\mathrm{Al}$ finger was assumed to be $10 \%$ of the SAW wavelength. This relatively large thickness was chosen in order to emphasize the reflection effects on SAW propagation. The material constants for GaAs and Al were extracted from Refs.14 and 15, respectively. The vertical scale in the figure is normalized to the frequency $\omega_{\mathrm{GaAs}}$ of the mode with wave vector $k_{\mathrm{SAW}} / 2$ on the bare GaAs substrate with $v_{\mathrm{GaAs}}=2866 \mathrm{~m} / \mathrm{s}$.

The IDT grating opens a stop band of relative width $\Delta \omega / \omega_{c}=0.05$ in the SAW dispersion (see Fig. 2). From the latter, we estimate an impedance ratio $F=\left(1-\Delta \omega / \omega_{c}\right) /(1$ $\left.+\Delta \omega / \omega_{c}\right) \approx 0.9$ and a maximum reflection coefficient per grating period $\left.r\right|_{\max } \lambda_{\text {IDT }}=|1-F| \approx 0.08$. According to Eq. (2), the strong reflection coefficient thus limits the number of periods contributing to the launched SAW mode to $L_{\text {eff }}=13$.

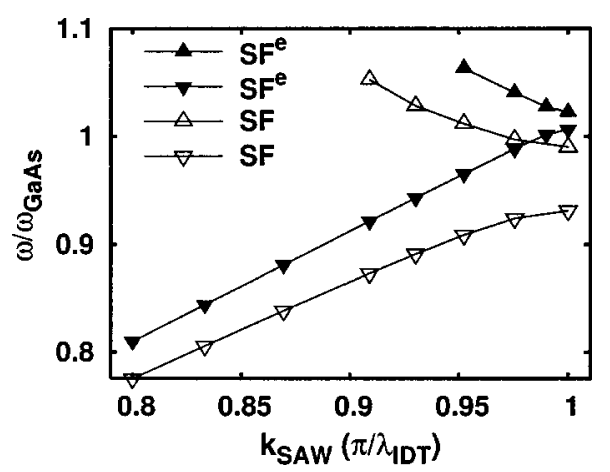

FIG. 2. Dispersion relation of SAW modes propagating on normal (open symbols) and embedded (solid symbols) aluminum gratings on GaAs for wave vectors $k$ close to the boundary $k=\pi / \lambda_{\text {IDT }}$ of the mini-Brillouin zone, where $\lambda_{\text {IDT }}$ denotes the grating period. The thickness of the Al film corresponds to $10 \%$ of the SAW wavelength. The angular frequencies are normalized to the average frequency of the stop-band $\omega_{\mathrm{GaAs}}=\pi v_{\mathrm{GaAs}} / \lambda_{\mathrm{IDT}}$, where $v_{\mathrm{GaAs}}=2866 \mathrm{~m} / \mathrm{s}$ denotes the SAW velocity on the bare substrate. The modes indicated by an upper triangle $(\triangle)$ have nonvanishing strain components $u_{x x}$ and $u_{z z}$, where $\mathbf{u}$ denotes the SAW displacement field and the coordinate system is defined in Fig. 1. The modes indicated by down triangles $(\nabla)$ are pure shear modes polarized in the $x z$ plane (i.e., only $u_{x z}$ $\neq 0)$.

Furthermore, the dispersion bends considerably close to the edge of the mini-Brillouin zone leading to modes with vanishing group velocities at $k=\pi / \lambda_{\text {IDT }}$.

The reduction of the acoustic reflection arising from the finger embedding becomes clear when similar calculations are performed for gratings buried into the substrate. The solid symbols in Fig. 2 show the dispersion relation for an embedded grating with the same metal thickness and dimensions as the one described above. The relative width of the stop band reduces to $\Delta \omega / \omega_{c}=0.016$, leading to a maximum reflection coefficient $\left.r\right|_{\max } \lambda_{\text {IDT }} \approx 0.04$ per period, which is considerably smaller than in the previous case. Note, in addition, that the flattening of the SAW dispersion near the stop band is much less pronounced than in the previous one.

The dependence of the stop band width on metallization thickness is displayed in Fig. 3. Note again that the splitting $\Delta \omega=\omega^{+}-\omega^{-}$between the modes $\omega^{+}$and $\omega^{-}$defining the stop band is considerably smaller in IDTs with embedded fingers. The small splitting is partially attributed to the vanishing

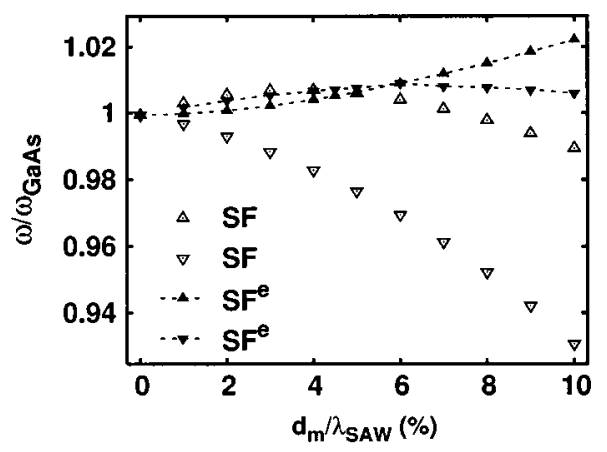

FIG. 3. Dependence of the frequency of the SAW modes $\omega^{+}$and $\omega^{-}$defining the stop band of SF IDT gratings on the thickness $d_{m}$ of the Al metallization. The open and solid symbols correspond to normal and embedded gratings, respectively. The frequencies are normalized to that of the mode with wave vector $\pi / \lambda_{\text {IDT }}$ on the bare GaAs substrate. The symmetry of the modes is described in the caption of Fig. 2. 

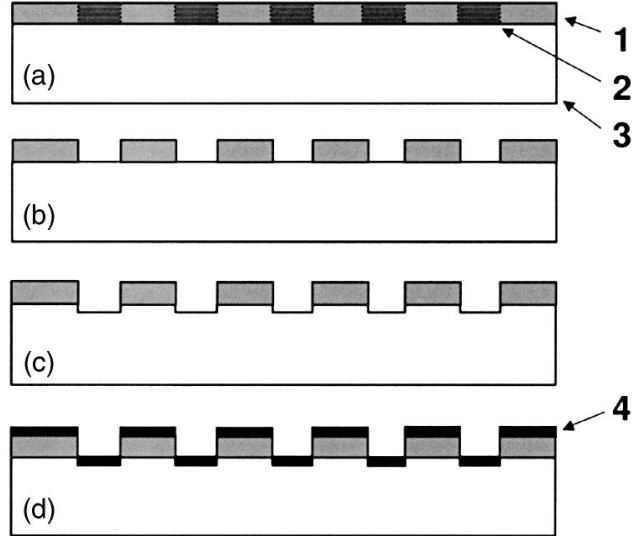

(e)

FIG. 4. Process steps for the fabrication of IDTs with embedded fingers: (a) photoresist (1) deposited on the surface of the substrate (3) for the definition of the metal finger areas (2) using photolithography; (b) development of the photoresist; (c) etching of the substrate; (d) deposition of the metal film (4); and (e) liftoff process to remove the excess metal.

stop band width for $d_{m} / \lambda_{\mathrm{SAW}} \approx 6 \%$. These results indicate that the finger reflection coefficient remains small in embedded SF IDTs over a wide range of metallization thicknesses (up to more than $6 \%$ of $\lambda_{\mathrm{SAW}}$ ).

A SAW Rayleigh propagating along a $\langle 110\rangle$ direction of the (001) GaAs substrate surface has nonvanishing strain components $u_{x x}=\partial u_{x} / \partial x$ and $u_{z z}=\partial u_{x} / \partial x$, and $u_{x z}=\frac{1}{2}\left(\partial u_{x} / \partial z\right.$ $\left.+\partial u_{z} / \partial x\right) \neq 0$. In these expressions, $\mathbf{u}$ denotes the SAW displacement field, and the coordinate system $(x, y, z)$ is defined in Fig. 1. The elastic eigenmodes generated by a SF IDT of infinite length, in contrast, are standing waves with a wave vector $k_{\mathrm{SAW}}=k_{\mathrm{IDT}} / 2$ exactly at the boundary of the miniBrillouin zone (cf. Fig. 2). The modes indicated by an upper triangle $(\triangle)$ in Figs. 2 and 3 have nonvanishing strain components $u_{x x}$ and $u_{z z}$ and negligible $u_{x z}$. The ones indicated by the down triangles $(\nabla)$, in contrast, are almost pure shear modes polarized in the $x z$ plane (i.e., only $u_{x z} \neq 0$ ). The grating thus splits the acoustic eigenmodes at the boundary of the Brillouin zone into a pure shear and a shearless wave. The SAW leaving the IDT is a linear combination of these modes: it is an evanescent wave within the IDT with a frequency lying within the stop band. Finally, the two zoneboundary modes cross for $d_{m} / \lambda_{\mathrm{SAW}} \approx 6 \%$, leading to a vanishing stop band width at this metallization coverage.

\section{EXPERIMENTAL DETAILS}

The IDTs with embedded fingers investigated here launch Rayleigh waves propagating along the [1피 direction of the GaAs (001) surface. They were fabricated on GaAs substrates following the procedure illustrated in Fig. 4, which involves five steps.

(a) Coating of the substrate (indicated by the number 2 in Fig. 4) with a positive photoresist (1) and subsequent definition of the metal finger areas (2) on the resist by photolithography.

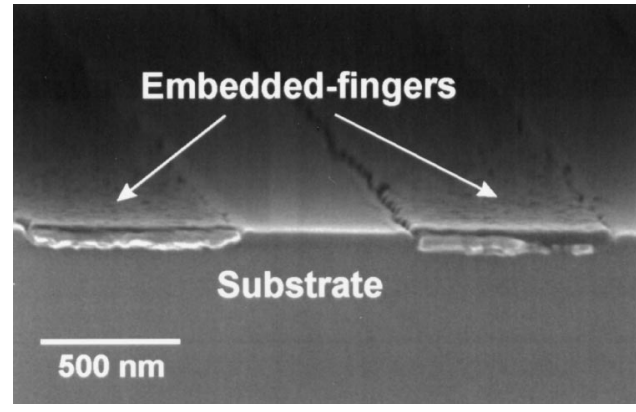

FIG. 5. Scanning electron micrograph of Al embedded fingers on a GaAs substrate.

(b) Development of the resist to expose the substrate areas underlying the metal.

(c) Etching of the finger areas using a $\mathrm{Cl}_{2}+\mathrm{N}_{2}$ plasmaetching process. The total pressure in the plasmaetching chamber was set to $2 \mathrm{~Pa}$ using flows of 1 and $20 \mathrm{SCCM}$ for $\mathrm{Cl}_{2}$ and $\mathrm{N}_{2}$, respectively. The excitation power used to generate the plasma was $50 \mathrm{~W}$ with a bias voltage of $136 \mathrm{~V}$. The nominal depth of the etching grooves was chosen to be equal to the thickness of the metallization layer $(\approx 60 \mathrm{~nm})$.

(d) Deposition of the metal film (4) consisting of a $\mathrm{Ti} / \mathrm{Al} / \mathrm{Ti}$ multilayer with thicknesses of 10, 40, and $10 \mathrm{~nm}$, respectively.

(e) Removal of the excess metal through a liftoff process.

Figure 5 displays the cross section of an embedded finger produced using the fabrication process described above. The highly directional plasma etching creates well-defined etch grooves with depth corresponding almost exactly to the thickness of the metallization film. In the fabrication, it is important to avoid the formation of grooves at the lateral interface between the embedded metal and the substrate, which efficiently scatter surface modes.

In order to evaluate the performance of the embeddedfinger concept, two sets of conventional and embeddedfinger IDTs (displayed in Table I) were fabricated with aluminum metal fingers of width and interspacing equal to $0.7 \mu \mathrm{m}$ Set 1 consists of double-finger (DF)—sometimes called also split-finger-IDTs designed for an operation at $\lambda_{\text {SAW }}=5.6 \mu \mathrm{m}$ and frequency $f_{\text {SAW }}=510 \mathrm{MHz}$. Set 2 is composed of single-finger IDTs for the generation of SAWs with $\lambda_{\text {SAW }}=2.8 \mu \mathrm{m}$ (operation frequency of $\approx 1.02 \mathrm{GHz}$ ). Each set includes a linear IDT (indicated by the subscript L) and two focusing IDTs with aperture angles $\phi$ of 0.1 and $0.2 \mathrm{rad}$ (denoted by the subscripts 1 and 2 , respectively). ${ }^{16}$ In the focusing devices, the fingers are curved in order to produce a converging SAW beam. Further details about the focusing IDTs can be found in Ref. 16

\section{RESULTS}

\section{A. Electrical characteristics}

The difference between IDTs with normal and embedded gratings becomes evident in their electric response. Figure 6 compares the rf reflection coefficient $s_{11}$ of embedded-finger $\left[\mathrm{SF}_{L}^{e},(\mathrm{a})\right]$ and conventional $\left[\mathrm{SF}_{L},(\mathrm{~b})\right]$ IDTs, as determined 
TABLE I. Depth of the electrical reflection coefficient $\left(\Delta\left|s_{11}\right|^{2}\right)$, electric efficiency $\left(\eta_{\mathrm{el}}\right)$, and acoustic conversion ratio $\left(\xi_{p}\right)$ for normal- and embedded-finger (e) IDTs. The IDT labels DF and SF indicate split- and single-finger structures, the subscripts $L$, and 1 and 2 denote linear IDTs and focusing IDTs with aperture angles $\phi$ of 0.1 and $0.2 \mathrm{rad}$, respectively.

\begin{tabular}{lccccccc}
\hline \hline & IDT & $\Delta\left|s_{11}\right|^{2}$ & $\Delta\left|s_{11}\right|^{2}(\mathrm{e})$ & $\eta_{\mathrm{el}}$ & $\eta_{\mathrm{el}}(\mathrm{e})$ & $\xi_{p}$ & $\xi_{p}(\mathrm{e})$ \\
\hline Set 1 with: & $\mathrm{DF}_{L}$ & $0.34 \pm 0.02$ & $0.30 \pm 0.02$ & $0.57 \pm 0.07$ & $0.28 \pm 0.01$ & 580 & $\ldots$ \\
$\lambda_{\mathrm{SAW}}=5.6 \mu \mathrm{m}$ & $\mathrm{DF}_{1}$ & $0.23 \pm 0.02$ & $0.23 \pm 0.02$ & $\ldots$ & $\ldots$ & 460 & $\ldots$ \\
$f_{\mathrm{SAW}}=510 \mathrm{MHz}$ & $\mathrm{DF}_{2}$ & $0.23 \pm 0.02$ & $0.19 \pm 0.02$ & $\ldots$ & $\ldots$ & 450 & $\ldots$ \\
& & & & & & & \\
Set 2 with: & $\mathrm{SF}_{L}$ & $0.73 \pm 0.02$ & $0.38 \pm 0.02$ & $0.030 \pm 0.002$ & $0.4 \pm 0.1$ & 170 & 900 \\
$\lambda_{\mathrm{SAW}}=2.8 \mu \mathrm{m}$ & $\mathrm{SF}_{1}$ & $0.60 \pm 0.02$ & $0.26 \pm 0.02$ & $\cdots$ & $\cdots$ & 25 & 580 \\
$f_{\mathrm{SAW}}=1.02 \mathrm{GHz}$ & $\mathrm{SF}_{2}$ & $0.19 \pm 0.02$ & $0.10 \pm 0.02$ & $\ldots$ & $\cdots$ & 31 & 360 \\
\hline \hline
\end{tabular}

using a microwave network analyzer. The frequency response $I(\omega)$ of a conventional IDT is given by $I(\omega)$ $\sim[\sin (x) / x]^{2}$, with $x=L_{\mathrm{IDT}} \pi / v_{G}$, where $v_{G}=\partial \omega_{\mathrm{SAW}} / \partial k \mathrm{de}-$ notes the SAW group velocity at the resonance frequency. ${ }^{17}$ The width of the resonance band of an IDT, defined as the frequency difference between the zeros of the $\sin (x) / x$ function, can be expressed as

$$
\Delta \omega_{\mathrm{SAW}}=\frac{2 \pi}{L_{\mathrm{IDT}}} v_{G} .
$$

For single-finger IDTs, $k_{\mathrm{SAW}}$ is located at the edges of the Brillouin zone, where, according to Fig. 2, $v_{G}$ decreases with increasing width of the stop bands, thus leading to a sharper resonance than in nonembedded IDTs.

The frequency response of the IDTs with embedded fingers show, in addition to the main resonance, a lower frequency side mode indicated by the arrows in Fig. 6. One is tempted to associate the main and side minima with the two modes defining the stop band (cf. Fig. 2). The separation between these modes, however, is approximately twice as large as the calculated stop-band width. In addition, by comparing embedded IDTs with different etch depths (and similar metallization thicknesses), we conclude that this frequency difference decreases with the stop-band width. We attributed the side mode to a secondary maximum of the $[\sin (x) / x]^{2}$ function, which is separated from the main one by a splitting $\Delta \omega_{\mathrm{SAW}}^{*}=\left(3 \pi / L_{\mathrm{IDT}}\right) v_{G}$. In the case of $\mathrm{SF}_{L}^{e}$ IDTs, in which the dispersion does not significantly deviate from a

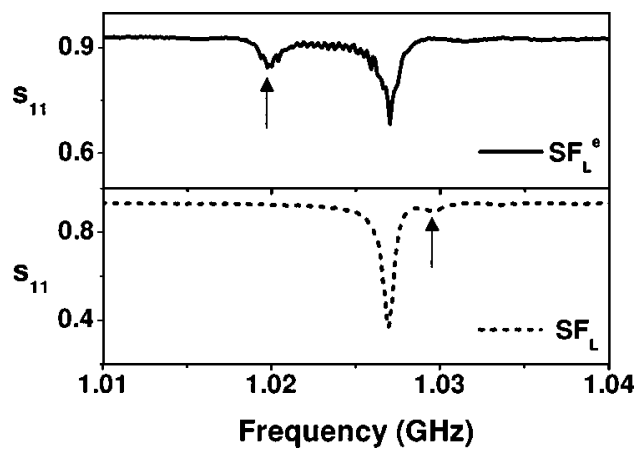

FIG. 6. rf reflection coefficient $s_{11}$ of $\operatorname{IDTs~}_{\mathrm{SF}_{L}^{e}}$ (a) and $\mathrm{SF}_{L}$ (b) determined using a network analyzer. The arrows indicate the first-order mode for each case. linear one, $v_{G} \sim v_{\mathrm{SAW}}$, leading to $\Delta \omega_{\mathrm{SAW}}^{*} /(2 \pi)=7.5 \mathrm{MHz}$ for $L_{\mathrm{IDT}}=560 \mu \mathrm{M}$. This value is very close to the measured splitting of $\Delta \omega_{\mathrm{SAW}}^{*} /(2 \pi)=7.3 \mathrm{MHz}$ in Fig. 6(a). Furthermore, $\Delta \omega_{\mathrm{SAW}}^{*} /(2 \pi)=2.5 \mathrm{MHz}$ is much smaller for $\mathrm{SF}_{L}$ IDTs, where the $v_{G}$ is reduced due to the wider stop band. Finally, we note that the side mode in nonetched IDTs is weaker and, in contrast with IDTs with embedded fingers, located on the high-frequency side of the main resonance. This behavior is probably associated with the different symmetries of the modes at the lower and higher frequency branches defining the stop band, as already discussed in connection with Fig. 2.

Table I compares, for the different transducer types, the depth of the electrical reflection coefficient $\Delta\left|s_{11}\right|^{2}=\left[s_{11}^{\text {(res) }}\right]^{2}$ $-\left[s_{11}^{(\text {off })}\right]^{2}$, where $s_{11}^{\text {(res) }}$ and $s_{11}^{\text {(off) }}$ denote the rf scattering reflection coefficient for the zero-order mode at the resonant frequency and off-resonance, respectively. $\Delta\left|s_{11}\right|^{2}$ corresponds to the fraction of the rf power applied to the IDT that is converted into acoustic modes. The differences in $\Delta\left|s_{11}\right|^{2}$ reflect the dependence of the electroacoustic coupling coefficients on the IDT geometry. As expected, the embedded gratings almost do not affect the $\Delta\left|s_{11}\right|^{2}$ parameter of DF IDTs. These transducers are characterized by a low SAW reflection coefficient, since Bragg reflection in this configuration is a second-order effect. For the SF structures, in contrast, $\Delta\left|s_{11}\right|^{2}$ decreases for the embedded fingers due to the strong reduction in the SAW reflection.

In spite of the smaller $\Delta\left|s_{11}\right|^{2}$, the reduced finger reflection also leads to a higher acoustic power outside the IDTs, a feature that can be verified by measuring the electrical transmission of a two-port delay line. In the absence of acoustic losses, the forward transmission scattering coefficient $s_{12}$ is related to the reflection coefficients $\left(s_{11}\right.$ and $\left.s_{22}\right)$ by the expression $\left|s_{12}\right|^{2}=\frac{1}{4} \Delta\left|s_{11}\right|^{2} \Delta\left|s_{22}\right|^{2}$, where the factor of 4 accounts for the bidirectionality of the IDTs. Table I also compares the electric efficiency $\eta_{\mathrm{el}}=4\left|s_{12}\right|^{2} /\left(\Delta\left|s_{11}\right|^{2} \Delta\left|s_{22}\right|^{2}\right)$ for different linear IDTs. The split-finger IDTs, in which no strong Bragg reflections are expected, have the highest $\eta_{\mathrm{el}}$ values (close to 0.6 ). The deviations of $\eta_{\mathrm{el}}$ from 1 in these IDTs is attributed to diffraction effects that spread the SAW beam and result in a deviation of its wave fronts from plane waves. $\eta_{\mathrm{el}}$ reduces by $\approx 50 \%$ in etched double-finger IDTs, probably due to inhomogeneities introduced by the etching process. The much smaller values of $\eta_{\mathrm{el}}$ in $\mathrm{SF}_{L}$ IDTs dem- 


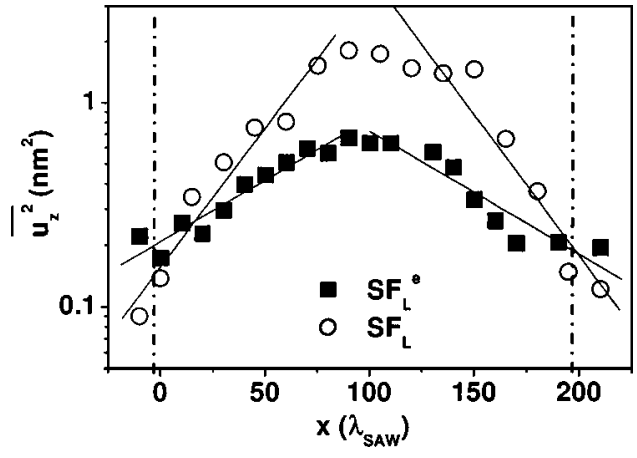

FIG. 7. Profiles of the squared vertical displacement $\overline{u_{z}^{2}}$ along the IDT length ( $x$ direction) of the linear $\mathrm{SF}_{L}^{e}$ and $\mathrm{SF}_{L}$ IDTs determined by interferometry. The dot-dashed lines at $x=0$ and $200 \lambda_{\text {SAW }}$ mark the edges of the IDTs.

onstrate that only a small fraction of the acoustic power generated by the emitting $\mathrm{SF}_{L}$ IDT reaches its counterpart at the opposite side of the delay line. The remaining power is dissipated through conversion into bulk modes upon reflection at the finger grating. The embedding process, however, increases $\eta_{\mathrm{el}}$ of $\mathrm{SF}_{L}^{e}$ by more than one order of magnitude to values comparable to the one in the $\mathrm{DF}_{L}$ structures. The $\eta_{\mathrm{el}}$ data for $\mathrm{SF}_{L}^{e}$ IDT displayed in Table I were obtained from measurements on 18 delay lines from four different wafers etched in distinct runs, thus attesting the reproducibility of the embedding process.

\section{B. Spatial distribution of the SAW fields}

The acoustic fields generated by the IDTs were probed using a microscopic Michelson interferometer to measure the vertical displacements induced by the SAW. The interferometer uses a single-mode laser source with a wavelength of $532 \mathrm{~nm}$, which is focused to an $\approx 1 \mu \mathrm{m}$ wide spot on the sample surface using a $50 \times$ objective. The laser spot can be scanned over the sample surface in order to measure spatially resolved profiles of the SAW amplitude. The output of the interferometer is detected by a fast photodiode (frequency response of $2 \mathrm{GHz}$ ). The amplitude of the diode signal, measured using a spectrum analyzer, is proportional to the square of the surface displacement field averaged in time $\overline{u_{z}^{2}}$ and, thus, to the local acoustic power density.

The superior performance of the IDTs with embedded fingers is demonstrated in Fig. 7, which compares $\overline{u_{z}^{2}}$ profiles measured along the length ( $x$ direction, as defined in Fig. 1) of the $\mathrm{SF}_{L}$ and $\mathrm{SF}_{L}^{e}$ IDTs. Both profiles were recorded by exciting the IDTs with the same nominal rf power $P_{\text {rf }}$ $=20 \mathrm{~mW}$. Although the $\mathrm{SF}_{L}^{e}$ IDT possess a much smaller $\Delta\left|s_{11}\right|^{2}$, the acoustic power delivered outside it (i.e., for $x<0$ and $x>200 \mu \mathrm{m}$ ) is higher than in the case of $\mathrm{SF}_{L}$ IDT. In the latter, most of the generated acoustic power is dissipated within the IDT. This result can be explained by the strong Bragg reflections at the metal grating for $\mathrm{SF}_{L}$, which turns this IDT into a standing wave cavity for the SAW beam with a high acoustic amplitude inside the transducer. In fact, the acoustic intensity $\overline{u_{z}^{2}}$ measured at the center of the finger area of these transducers is $\approx 20$ times larger than at the output (see Fig. 7). The multiple reflections also increase the scattering of the SAW into bulk modes, thus reducing $\overline{u_{z}^{2}}$ outside the IDT. The reflection effects are considerably reduced when the metal grating is embedded into the substrate $\left(\mathrm{SF}_{L}^{e}\right.$ IDT). The ratio between the $\overline{u_{z}^{2}}$ values in the middle and outside these IDTs reduce to $\approx 3$.

In both types of IDTs in Fig. 7, $\overline{u_{z}^{2}}$ decays exponentially towards the edges of the IDTs. The solid line are fits according to Eq. (2), which yield decay lengths $L_{\mathrm{dec}}$ of $\approx 30 \lambda_{\mathrm{SAW}}$ and $70 \lambda_{\text {SAW }}$ for IDTs with normal and embedded fingers, respectively. Using the simple model of Sec. II B, we estimate using $d_{m} / \lambda_{\mathrm{SAW}}=2.1 \%$ a lower limit for $L_{\text {eff }}$ equal to $9 \lambda_{\text {SAW }}$ for the normal SF IDT. A more realistic estimation can be obtained using the model outlined in Ref. 9. Using particle velocities $v_{x}=2.15 \mathrm{~m} / \mathrm{s}$ and $v_{z}=3.35 \mathrm{~m} / \mathrm{s}$ (keeping the coordinate notation defined in Fig. 1) calculated employing the simulation procedure described in Ref. 6 for an acoustic power density of $100 \mathrm{~W} / \mathrm{m}$, this model yields $L_{\text {eff }}$ $=23 \lambda_{\text {SAW }}$. This value compares well with the measured decay length for the SF IDT in Fig. 7.

\section{Focusing performance}

The acoustic SAW power delivered by the IDT can be determined by integrating the squared vertical displacement field $\overline{u_{z}^{2}}(y)$ determined by interferometry across the beam width ( $y$ direction). We use this fact to determine the acoustic conversion ratio $\xi_{p}$ (shown in Table I) defined as

$$
\xi_{p}=\frac{f_{\mathrm{SAW}}}{1 \mathrm{GHz}} \frac{1}{\Delta\left|s_{11}\right|^{2} P_{\mathrm{rf}}} \int_{-\infty}^{\infty} \overline{u_{z}^{2}}(y) d y,
$$

where the integral along the beam cross section is determined from $u_{z}$ measurements outside the IDT. $\xi_{p}$ quantifies the ability of a transducer to convert the applied rf power into a propagating SAW mode outside it. The $\Delta\left|s_{11}\right|^{2}$ factor corrects for the differences in the electric to acoustic conversion efficiencies arising from the different IDT geometries. The frequency ratio $f_{\mathrm{SAW}} /(1 \mathrm{GHz})$ was introduced in Eq. (4) in order to allow for the comparison of transducers with different operation frequency. This ratio takes into account the fact that for a fixed acoustic power density the squared amplitude of the SAW field is inversely proportional to the operation frequency.

The data for the linear IDTs show again the improvement in the conversion efficiency in IDTs with embedded fingers, which becomes comparable to those measured in DF IDTs. The performance of focusing IDTs is also considerably improved when the devices are fabricated with embedded gratings. As mentioned previously, these IDTs have curved fingers with a shape following curves of constant SAW group velocity. ${ }^{16}$ Due to the acoustic anisotropy inherent of crystalline materials, the design of focusing IDTs relies on the precise knowledge of the dependence of the SAW properties on the propagation direction. ${ }^{16}$ In SF IDTs, the latter also depends sensitively on the dimensions of the metal grating (Fig. 2). Small fluctuations in the thickness or width of the fingers lead, therefore, to considerable variations in focusing performance. This effect adds to the losses induced by acoustic reflection at the fingers, leading to conversion ratios $\xi_{p}$ considerably lower than for split-finger IDTs (see Table I). In contrast, when the IDT fingers are embedded into the sub- 
strate the grating effects on the SAW dispersion are significantly reduced. As a result, $\xi_{p}$ of focusing IDTs becomes enhanced by more than one order of magnitude, thus leading to performances comparable to the ones of split-finger IDTs.

\section{CONCLUSIONS}

We have investigated single-finger IDTs, which substantially reduce the Bragg reflection of the SAW on the IDT metal grating by embedding the metal grating into the substrate. We have shown that this procedure also considerably reduces the effects of the metal grating on the SAW propagation properties (such as the phase and group velocity as well as their dependence on the propagation direction). IDTs with embedded gratings are particularly interesting for the generation of high-frequency SAWs on weak piezoelectric substrates (such as, for instance, the conventional III-V semiconductors), where the acoustic reflections at the long metal grating limit the maximum acoustic field that can be generated by the IDT.

We have demonstrated the superior performance obtained by embedding the fingers through calculations as well as through a direct experimental comparison of the acoustic power delivered by normal and embedded-finger IDTs. For long IDTs, the acoustic field intensities delivered by the new structures are at least a factor of 4 larger than in conventional IDTs. Furthermore, we show that the weaker impact of the embedded grating on SAW propagation is particularly important for focusing IDTs, where the focusing performance depends crucially on the propagation properties. The use of embedded gratings in this case leads to a significant enhancement in the acoustic power delivered by the devices.

\section{ACKNOWLEDGMENTS}

The authors thank H. T. Grahn for comments and for a critical reading of the manuscript. They acknowledge the technical expertise of $\mathrm{S}$. Krauß in the preparation of the samples and of A. Bluhm in the SEM measurement. Partial support from the Deutsche Forschungsgemeinschaft (Project No. SA598/3-1) and the Deutscher Akademischer Austauschdienst are gratefully acknowledged.

${ }^{1}$ M. J. Hoskins, H. Morkoç, and B. J. Hunsinger, Appl. Phys. Lett. 41, 332 (1982).

${ }^{2}$ C. Rocke, S. Zimmermann, A. Wixforth, J. P. Kotthaus, G. Böhm, and G. Weimann, Phys. Rev. Lett. 78, 4099 (1997).

${ }^{3}$ P. V. Santos, J. Appl. Phys. 89, 5060 (2001).

${ }^{4}$ M. M. de Lima, Jr., R. Hey, and P. V. Santos, Appl. Phys. Lett. 83, 2997 (2003).

${ }^{5}$ F. W. Beil, A. Wixforth, and R. H. Blick, Physica E (Amsterdam) 13, 473 (2002).

${ }^{6}$ Y. Takagaki, E. Wiebicke, P. V. Santos, R. Hey, and K. H. Ploog, Semicond. Sci. Technol. 17, 1008 (2002).

${ }^{7}$ S. Rooth, Patent No. 6,268,680 B1, 2001.

${ }^{8}$ D. Royer and E. Dieulesaint, Elastic Waves in Solids (Springer, Heidelberg, 2000).

${ }^{9}$ S. Datta and B. J. Hunsinger, J. Appl. Phys. 50, 5661 (1979).

${ }^{10} \mathrm{~S}$. Rooth and A. Rønnekleiv, in Proceedings of the 1995 IEEE Ultrasonics Symposium, edited by U. Efron (IEEE, Seattle, 1995), pp. 281-285.

${ }^{11}$ B. A. Auld, Acoustic Fields and Waves in Solids (Robert E. Krieger, Malabar, FL, 1990).

${ }^{12}$ T. Aono and S. Tamura, Phys. Rev. B 58, 4838 (1998).

${ }^{13}$ Y. Tanaka and S. Tamura, Phys. Rev. B 58, 7958 (1998).

${ }^{14}$ F. Jungnickel, S. Makarov, E. Chilla, and H.-J. Fröhlich, Sens. Actuators, A 61, 313 (1997).

${ }^{15}$ A. J. Slobodnik, E. D. Conway, and R. T. Delmonico, Microwave Acoustics Handbook, Vol. 3: Bulk Wave Velocities (National Technical Information Service, US Department of Commerce, Springfield, VA, 1973).

${ }^{16}$ M. M. de Lima Jr., F. Alsina, W. Seidel, and P. V. Santos, J. Appl. Phys. 94, 7848 (2003)

${ }^{17}$ S. Datta, Acoustic Surface Wave Devices (Prentice Hall, London, 1986). 